\title{
FACTORES QUE AFECTAN LA VARIACIÓN DE LOS INGRESOS FOB POR EXPORTACIÓN DE BANANO Y PLÁTANO ECUATORIANO
}

\author{
FACTORS AFFECTIMG THE VARIATION IN FOB INCOME \\ FROM EXPORTS OF ECUADORIAN BANANAS AND PLATAINS
}

\author{
Danny Trujillo Sandoval' Sebastián Pereira Ordóñez² Génesis Torres Cabrera
}

\begin{abstract}
Resumen
Las exportaciones de materias primas en los paises latinoamericanos constituyen una fuente fundamental de ingresos para atender sus presupuestos. El banano y plátano son parte de los productos que el Ecuador más exporta a nivel mundial, razón por la cual, la variación de los ingresos por este rubro afecta de manera directa al ingreso de divisas en la economía nacional. El objetivo de la presente investigación consiste en la determinación de los factores que inciden en esta variación, a través del análisis y la descomposición econométrica de la serie temporal de exportación de banano y plátano ecuatoriano en el período 2013 -2017. Como resultado de la descomposición se ha obtenido e identificado los picos negativos y positivos del componente irregular y estos, a su vez, han sido categorizados de acuerdo a un análisis de entorno empresarial, con lo que, finalmente, se ha logrado definir los factores claves que influyen en la variación de los ingresos FOB por exportación de banano y plátano ecuatoriano. Los resultados obtenidos son relevantes, ya que se convierten en un insumo para que las instituciones responsables de la generación de políticas públicas protejan y mejoren la competitividad de estos productos en los mercados internacionales.
\end{abstract}

\section{Palabras clave}

Banano ecuatoriano, exportaciones, series temporales, econometría.

JEL: F13

\begin{abstract}
Exports of raw materials are a fundamental source of income in Latin American countries. Banana and plantain are two of the products that Ecuador exports the most worldwide, which is why the variation in income from these items directly affects the entry of foreign currency into the national economy. The objective of this research is to determine the factors that influence this income variation through analysis and econometric decomposition of the Ecuadorian banana and plantain export time series in the period 2013-2017; as a result of the decomposition, the negative and positive peaks of the irregular component have been obtained and identified; and, these in turn have been categorized according to an analysis of the business environment, with has led to define the key factors that influence the variation in FOB income from the export of Ecuadorian bananas and plantains. The results obtained are relevant since they are an input for the institutions responsible for the generation of public policies to protect and improve the competitiveness of these products in international markets.
\end{abstract}

\section{Keywords}

Ecuadorian banana, exports, time series, econometrics

JEL: F13

\footnotetext{
1 Consultor-capacitador Independiente, Quito - Ecuador (dannyts@hotmail.com).

$2 \quad$ Universidad Internacional SEK, Quito - Ecuador (sebastian.pereira@uisek.edu.ec)

3 Universidad Internacional SEK, Quito - Ecuador (gtorres.cea@uisek.edu.ec)
} 


\section{Introducción}

La dinámica e importancia del comercio internacional radica en las importaciones y exportaciones de productos y servicios de diferentes países y regiones del mundo. El Ecuador es un país que se caracteriza por mantener a flote su economía, a través de los ingresos provenientes de la exportación de materias primas, entre ellas: el cacao, el banano y las flores (Camino Mogro, Andrade Díaz y Pesántez Villacís, 2016).

El banano es uno de los productos que Ecuador más exporta a nivel mundial, su volumen representa alrededor del $25 \%$ de las exportaciones totales en el mundo (The Observatory of Economic Complexity, 2020). Tan solo en la Unión Europea su exportación es alrededor del 40 \% (Vásquez Orozco, 2010). Además, dentro de las exportaciones ecuatorianas es el segundo rubro de mayor exportación (Jiménez Cabrera, 2018).

Los principales productores de banano son África, Brasil, Colombia, China, Costa Rica, Ecuador, Filipinas, Guatemala, Honduras, India, Indonesia, Panamá, República Dominicana y Tailandia, ya que sus economías dependen en gran medida de la agricultura (FAO, 2018). En la economía del banano se puede observar una gran concentración en el mercado mundial, tanto por quienes exportan la fruta como por quienes la importan; de esta forma, los principales importadores de banano son países como Estados Unidos, Alemania y Bélgica. Pero se puede ver una creciente importación de banano en países asiáticos como China y Japón, lo que hace que el mercado sea más grande y por consiguiente más competitivo para los productores de banano.

De todos los países importadores relevantes, el único que tiene procesos regulatorios sobre la importación es la Unión Europea, lo que hace que el precio del banano sea en promedio 80 \% más elevado que en Esta- dos Unidos. El $63 \%$ del volumen de banano comercializado en la Unión Europea tiene su origen en los países latinoamericanos que tienen influencia directa del dólar estadounidense en sus economías, el $17 \%$ en países de las regiones de África, Caribe y el Pacífico, y el restante $20 \%$ es de producción propia en Canarias, Madeira, Creta, y en Guadalupe y Martinica (Martínez y Peña, 2005).

Debido a la globalización de los mercados, los países exportadores de banano afinan sus estrategias de comercialización para lograr ventajas comparativas en los mercados internacionales (Camino Mogro et al., 2016). Muchos son los factores que los productores toman en cuenta para incrementar sus volúmenes de exportación: políticas de subsidios e impuestos, facilidad en trámites de exportación, programas de incremento de productividad, programas de control de plagas, capacitaciones técnicas, entre otros. Es decir, los volúmenes exportados y sus correspondientes ingresos van a depender de muchos factores, que pueden ser políticos, económicos, sociales, ambientales y/o tecnológicos.

Existen algunas prácticas que están arraigándose en países productores para que su banano sea más atractivo para el consumidor, algunas de estas prácticas se basan en el manejo y control de la cadena de valor (Robinson, 2010). El enfoque en la cadena de valor se da sobre una propuesta cuyo principal objetivo consiste en mejorar las condiciones de trabajo de los productores y eliminar el uso de químicos o pesticidas, ya que pueden tener repercusiones negativas tanto en seres humanos como en organismos no humanos dentro y fuera de las plantaciones (Sanderson Bellamy, 2012).

Es importante recalcar que, a más de factores relacionados con la cadena de valor, la calidad del banano constituye uno de los elementos decisores para su importación y exportación; al respecto, el Ecuador 
es el mayor exportador con un producto que cumple los más altos estándares de calidad, cuya producción ha alcanzado estándares internacionales de protección ambiental (Brassel, Breilh y Zapat, 2011).

Otro factor a tomar en cuenta es el clima, ya que si existen problemas como cambios de temperatura, inundaciones o sequías, se verán afectadas las plantaciones y por ende los volúmenes de producción, los precios y la actividad comercial.

De acuerdo a cifras del Banco Central del Ecuador, los ingresos FOB por exportación de banano y plátano en el año 2019 representaron el $18.49 \%$ del total de los productos primarios exportados y el $14.76 \%$ del total de los ingresos percibidos por exportaciones, en los que se incluye productos primarios e industrializados (Banco Central del Ecuador, 2020); razón por la que el banano constituye uno de los ejes de la economía de Ecuador, de tal manera que reducidos volúmenes de exportación afectan de manera negativa a la economía nacional (Fierro Ulloa y Villacres Rojas, 2014).

Por lo expuesto, es relevante el análisis del comportamiento de los ingresos generados por las exportaciones de banano (ingresos FOB), ya que, como se ha mencionado existen varios factores que influyen directa 0 indirectamente en los volúmenes de exportación y de manera subsecuente en los ingresos monetarios, que revelan su importancia en el desarrollo económico social de la población.

Por otro lado, es importante recalcar que los ciclos económicos, caracterizados por períodos de contracción y expansión, afectan directamente a la caja fiscal de los países, generando picos positivos y negativos de comportamiento en la actividad exportable, por lo que resulta necesario estudiar los factores que los generan, y entenderlos es clave en el desarrollo sostenible.

El objetivo de la presente investigación consiste en determinar aquellos factores que inciden de manera significativa en la variación de los ingresos FOB por exportaciones ecuatorianas de banano y plátano, a través de herramientas de análisis de series de tiempo, a fin de proporcionar información relevante, que permita direccionar esfuerzos en cuanto a la generación de políticas públicas que incentiven el desarrollo de este sector productivo, considerado como el más importante en rubros de exportación del Ecuador.

\section{Balanza comercial ecuatoriana}

Según información oficial del Banco Central del Ecuador, la balanza comercial total del Ecuador en el año 2017 fue superavitaria, con aproximadamente 89.2 millones de dólares; esto como consecuencia de la recuperación en valores FOB de las exportaciones petroleras y crecimiento de las no petroleras. Sin embargo, en el caso de la balanza no petrolera se registró un aumento en su déficit (110.5\%) frente al valor registrado en el año 2016. (Banco Central del Ecuador, 2018).

Las exportaciones no petroleras totales en el año 2017 registraron un crecimiento del 7.7 \% en comparación con el 2016. Para el caso de las exportaciones no petroleras tradicionales se registró un incremento del $10.3 \%$. Las participaciones de los productos tradicionales y no tradicionales en las exportaciones no petroleras registradas en 2017 fueron de $58.3 \%$ y $41.7 \%$, respectivamente. Dentro de las exportaciones no petroleras tradicionales, las participaciones de los principales productos con respecto al total de exportaciones no petroleras fueron: banano y plátano (24.9\%), camarón (24.9\%), cacao y elaborados (5.6\%), atún y pescado (2\%), y, café y elaborados (1\%). (Banco Central del Ecuador, 2018).

Ecuador es el mayor productor y exportador de banano del mundo, cuenta con un promedio anual de ventas extranjeras de alrededor de 6 millones de toneladas 
métricas comercializadas en los diferentes continentes. (León Serrano et al., 2020), resultado de varios factores que responden al fortalecimiento de ventajas comparativas relacionadas a la producción, estrategias empresariales, recursos y tecnología (Vásquez Orozco, 2010).

También es importante mencionar que la estructura de ingresos del Ecuador tiene como principales fuentes de financiamiento de su presupuesto a las exportaciones, fundamentalmente de petróleo y sus derivados, el financiamiento internacional y la recaudación tributaria (De la Cruz Guerrero y Rosales Nieto, 2016). A pesar de lo mencionado, los ingresos por exportaciones de banano a más de generar ingresos o divisas a la economía ecuatoriana, permite la generación de fuentes de empleo a nivel intensivo, lo que se apalanca con las condiciones climáticas y ubicación geográfica que favorecen a la producción durante todo el año.

A partir del año 2014 hasta el año 2017 las exportaciones no petroleras ecuatorianas comienzan a dominar en porcentaje su participación en las exportaciones totales del país como se puede observar en la figura 1 , esto agregado a que el petróleo es un bien no renovable, genera la necesidad de perspectivas futuras sin dependencia de petróleo y que incentiven la participación mayoritaria de exportaciones no petroleras.

Figura 1. Participación porcentual sobre el total de las exportaciones

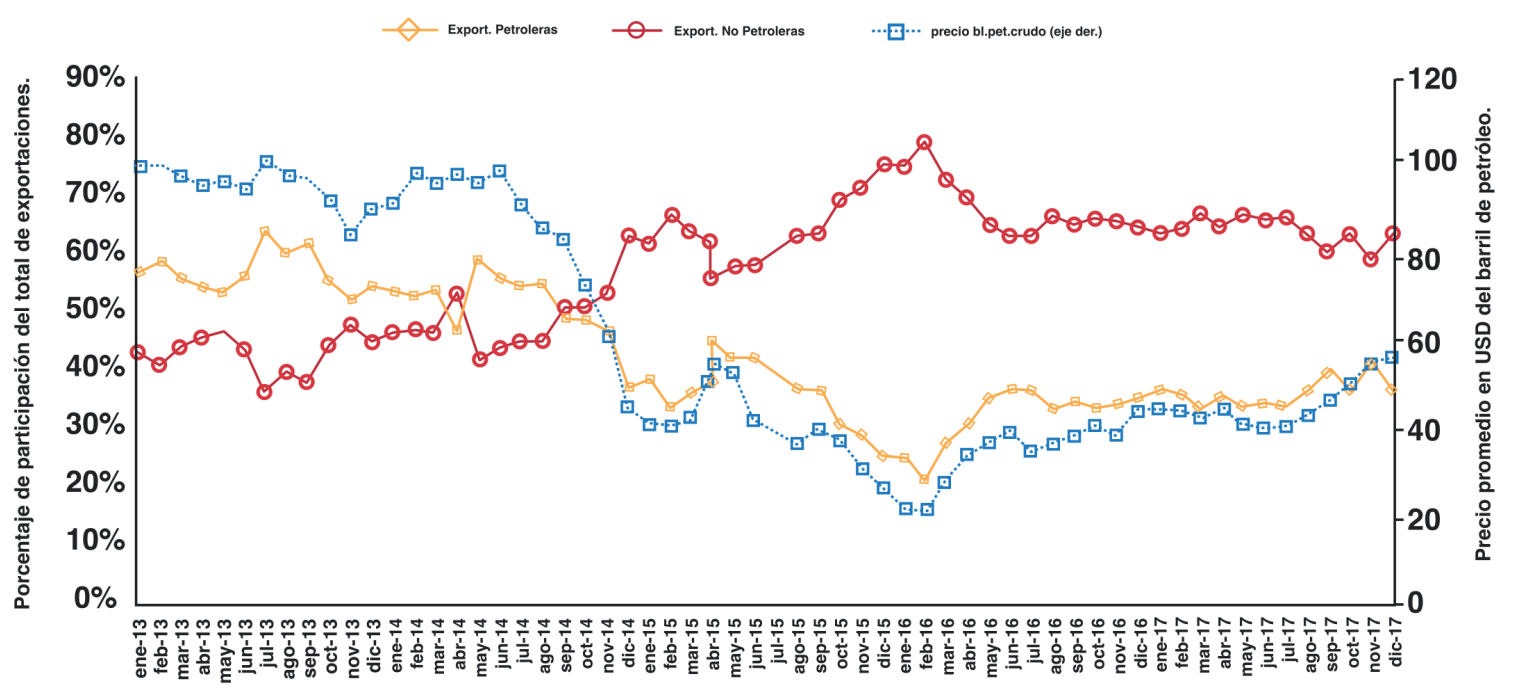

Fuente: Banco Central del Ecuador (2018)

\section{Destino de las exportaciones de banano y plátano ecuatoriano}

Como se mencionó anteriormente dentro de las exportaciones no petroleras ecuatorianas, el principal producto es el banano y plátano para el año 2017 y en dicho caso los principales destinos de exportación fueron Rusia con $21.1 \%$, Estados Unidos con $17.2 \%$,
Italia con 8.8 \% y Alemania con $8.6 \%$. (Banco Central del Ecuador, 2018).

El banano ecuatoriano posee características atractivas en cuanto a sabor y calidad es por ello que es competitivo a nivel de mercados internacionales y ha logrado 
apertura en el bloque europeo (Andrade Rodríguez y Meza Lino, 2017).

Los volúmenes de las exportaciones de materias primas y sus respectivos ingresos han sido sujetos de varios estudios analizar, el porqué de su incremento o reducción a fin de definir su grado de afectación en el crecimiento económico y para realizar proyecciones o pronósticos para la toma de decisiones (Trujillo, Apunte y Pereira, 2019). Con los escenarios planteados resulta necesario contar con información de mercados, estudios de factores incidentes y análisis de las diferentes variables, con el fin de aportar a las transacciones dentro de la economía ecuatoriana.

\section{Series de tiempo y exportaciones}

Los ingresos por exportaciones de cualquier bien, producto o servicio se registran como un valor en diferentes momentos. Son estos valores en su conjunto los que se definen como una serie de tiempo (Gujarati, 2007).

Una serie de tiempo es un grupo de datos registrados durante un periodo semanal, trimestral o anual. (Lind, Marchal y Wathen, 2012)

Para el caso de series de tiempo asociadas a exportaciones, los valores a utilizar podrían ser FOB, toneladas métricas, etc., dependiendo el tipo de estudio y variables asociadas.

\section{Descomposición de las series de tiempo}

Una serie de tiempo puede ser descompuesta en sus componentes a fin de analizar a profundidad su comportamiento y los factores que la afectan. La descomposición clásica de una serie de tiempo es un método que tiene como base el supuesto de que la serie de datos se puede desagregar en componentes como: tendencia, ciclo, estacionalidad e irregularidad; que se describen a continuación: a) Tendencia.- Una serie de tiempo con tendencia es aquella que contie- ne un componente de largo plazo que representa el crecimiento o declinación de la serie a través de un período amplio. b) Estacional.- Se define como estacional una serie de tiempo con un patrón de cambio a sí mismo año tras año. Por lo regular, el desarrollo de una técnica de pronóstico estacional comprende la selección de un método multiplicativo o uno de adición y estimar después indices estacionales a partir de la historia de la serie. c) Ciclo.- El efecto cíclico se define como la fluctuación en forma de onda alrededor de la tendencia. Los patrones cíclicos tienden a repetirse en los datos cada dos, tres o más años, dependiendo de la naturaleza de la serie analizada. d) Irregular.- El componente irregular de la serie de tiempo es el factor residual, es decir, "todo lo que sobra" y toma en consideración las desviaciones de los valores reales de la serie de tiempo en comparación con los esperados; es el elemento aleatorio (Murillo, Trejos y Carvajal Olaya, 2003).

Con el objeto de analizar una serie de tiempo y a su vez estimar un valor futuro de la variable de estudio, existen dos tipos de modelos de métodos cuantitativos que agrupan datos, que son los univariados y los causales.

Los modelos univariados predicen el futuro de una serie en función de su comportamiento histórico propio; son muy útiles si el patrón detectado en el pasado se mantiene hacia el futuro, de lo contrario no son aconsejables (Moreno Sarmiento, 2013).

\section{Metodología}

El presente estudio es parte de un proyecto de investigación, por lo cual se aplica la metodología presentada por Trujillo et al. (2019), con todos sus elementos asociados como el diseño, instrumentos y procedimiento. En el presente documento se aplica a la serie temporal 2013-2017 del banano y plátano de exportaciones FOB - Ecuador, con el fin de iden- 
tificar los factores que afectan a la variación en ingresos FOB al Estado ecuatoriano.

\section{Diseño de la investigación}

El diseño de la investigación se fundamenta en una metodología mixta compuesta por dos fases secuenciales. La primera consiste en una investigación cuantitativa no experimental, con temporalidad longitudinal y alcance explicativo. La segunda fase consiste en la aplicación de investigación cualitativa basada en modalidad y revisión documental, que permite el relevamiento y la identificación de factores que inciden en la variación de ingresos FOB, por exportaciones ecuatorianas de banano y plátano.

\section{Recolección de datos e instrumentos}

En el presente estudio se compilaron datos oficiales y depurados de ingresos FOB por exportaciones ecuatorianas de banano y plátano, correspondientes al período comprendido entre los años 2013 y 2017, obtenidos a través del Banco Central del Ecuador.

$$
Y_{t}=T_{t}+S_{t}+I_{t}
$$

Donde $Y_{t}$ representa el valor total del período $t, T_{t}$ el componente de tendencia l del pe-

$$
\begin{aligned}
& M A D=\frac{1}{n} \sum_{t=1}^{n}\left|Y_{t}-\hat{Y}_{t}\right| \\
& M S E=\frac{1}{n} \sum_{t=1}^{n}\left(Y_{t}-\hat{Y}_{t}\right)^{2} \\
& R M S E=\sqrt{\frac{1}{n} \sum_{t=1}^{n}\left(Y_{t}-\hat{Y}_{t}\right)^{2}} \\
& M A P E=\frac{1}{n} \sum_{t=1}^{n} \frac{\left|Y_{t}-\hat{Y}_{t}\right|}{\left|Y_{t}\right|} \\
& M P E=\frac{1}{n} \sum_{t=1}^{n} \frac{\left(Y_{t}-\hat{Y}_{t}\right)}{Y_{t}}
\end{aligned}
$$

Para el tratamiento de la información correspondiente a una serie temporal se procedió a realizar su descomposición econométrica, a través del software estadístico R-project versión 3.4.2 con el entorno de trabajo RStudio versión 1.1.442 y se complementó con Matlab 2018b y Excel para el desarrollo de gráficas.

\section{Procedimiento de análisis de datos}

Para la primera fase, en el entorno de trabajo de RStudio se realizó la descomposición econométrica de la serie temporal de ingresos FOB por exportaciones de banano y plátano, en sus componentes: de tendencia, estacional e irregular, con la aplicación del método STL, presentado por Cleveland (1990), en la cual la variable corresponde a los valores mensuales de ingresos FOB y seleccionando la forma aditiva, de acuerdo con la ecuación 1 (Hanke y Wichern, 2010); posteriormente se validó la descomposición econométrica con los índices estadísticos de ajuste MAD, MSE, RMSE, MAPE Y MPE, acorde a las ecuaciones 2 a 6 (Hanke y Wichern, 2010).

ríodo t, $S_{t}$ el componente estacional l del período t e $I_{t}$ el componente irregular I del período $t$. 
En las ecuaciones 2 a $6 Y_{t}$ representa el valor real total del período t, $\hat{Y}_{t}$ el valor pronosticado en el período $t$ y $n$ la cantidad de valores de la serie temporal.

Una vez validada la descomposición, se separó el componente irregular de la serie temporal y se filtraron los picos negativos y los picos positivos, creando un subgrupo para cada uno. Después, se aplicó estadística descriptiva para generar medidas de máximos, mínimos, primer cuartil Q1, segundo cuartil Q2 y tercer cuartil Q3.

Posteriormente, se procedió a realizar un nuevo filtro con los valores superiores al segundo cuartil Q2, en cada subgrupo de picos; con esto se organizaron los picos resultantes para dar paso a la segunda fase que, como se comentó con anterioridad, fue cualitativa. En esta se utilizaron los picos resultantes de la fase anterior, los cuales fueron analizados de manera individual, a través de revisión documental, a fin de identificar las causas o factores que generaron las variaciones en la serie temporal.

Una vez identificadas las referidas causas, se procedió a clasificarlas a través de un análisis de entorno empresarial (PES-
TEL). Con los resultados obtenidos se procedió a la construcción de diagramas de Pareto para cada subgrupo de picos, identificando las zonas vitales y triviales, así como también los factores clave que incidieron en las variaciones de ingresos FOB por exportaciones de banano y plátano, en el período comprendido entre los años 2013 y 2017.

\section{Análisis de resultados}

\section{Descomposición econométrica de la serie temporal}

Se realiza la descomposición econométrica de la serie temporal de ingresos FOB para exportaciones de banano y plátano ecuatoriano, con la aplicación del método STL presentado por (Cleveland, 1990), y seleccionando la forma aditiva; esto conforme a las características naturales de la serie temporal. Se ha utilizado R-project versión 3.4.2 mediante la herramienta RStudio versión 1.1.442 para la descomposición y Matlab 2018b para la elaboración de gráficas.

En la figura 2 se pueden observar las gráficas resultantes.

Figura 2. Componentes de la serie de tiempo

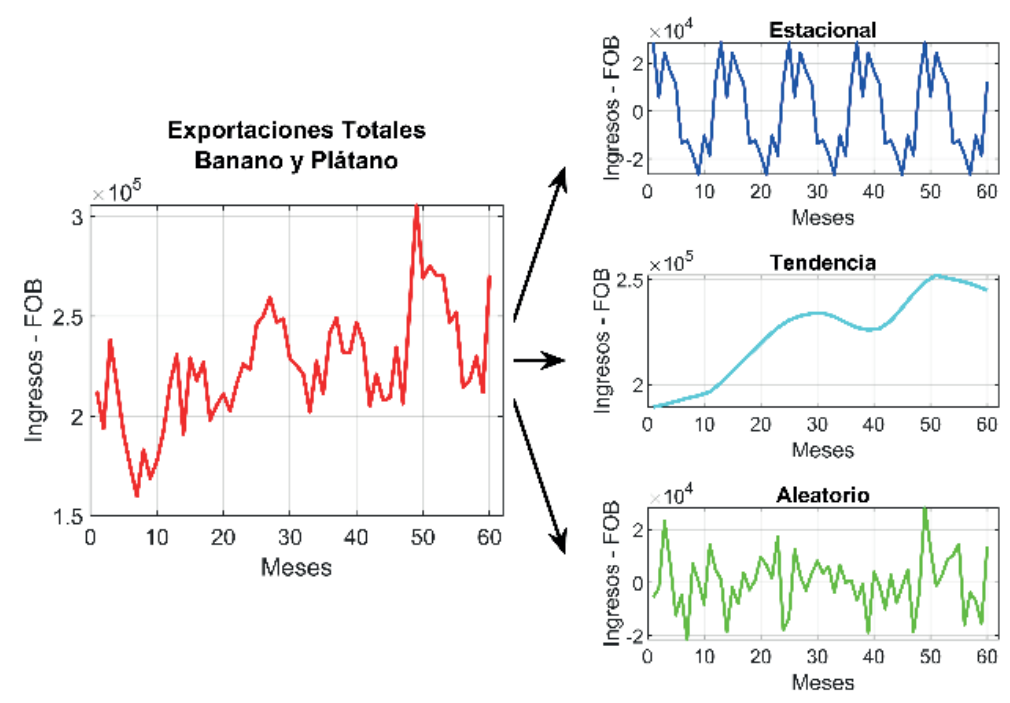

Fuente: elaboración propia. 
Como se puede observar en la figura 2, los componentes son de tendencia estacional e irregular, esta último será sujeto de estudio, pues se representa la aleatoriedad existente en ingresos FOB por exportaciones de banano y plátano al Estado ecuatoriano. Dicho estudio permite sentar bases para controlar tal variabilidad y poder mantener y planificar los ingresos a futuro.

\section{Índices de ajuste de la descomposición}

Una vez descompuesta la serie temporal es necesario validarla con indicadores de ajuste. que permitan confirmar que la descomposición es adecuada y se puede continuar con el estudio de uno o varios de los componentes resultantes.

Tabla 1. Índices de ajuste de la descomposición

\begin{tabular}{|l|c|}
\hline \multicolumn{1}{|c|}{ Índices } & Valor para STL \\
\hline Desviación media absoluta (MAD). & 7996.28 \\
\hline Error cuadrático medio (MSE). & 107074496.16 \\
\hline Raíz cuadrada del error cuadrado medio (RMSE). & 10347.68 \\
\hline Error porcentual absoluto medio (MAPE). & 0.03610 \\
\hline Error porcentual medio (MPE). & -0.00186 \\
\hline
\end{tabular}

Fuente: elaboración propia.

Como se puede observar en la tabla 1, los indicadores de ajuste son adecuados, pues se debe recordar que las exportaciones para el período de estudio tienen valores FOB de cientos de miles de dólares, por lo cual una desviación media absoluta de 7996.28 es baja, y los errores porcentual absoluto medio con $3.6 \%$ y un porcentual medio de $-0.1 \%$ representan valores bastante bajos y adecuados, del ajuste de la descomposición para el presente proyecto.

\section{Análisis del componente aleatorio}

Con la validación exitosa de la descomposición econométrica, el elemento sujeto de estudio seleccionado es el componente aleatorio. En esta sección se da tratamiento a ello y se aplican criterios estadísticos descriptivos con el fin de identificar los valores claves de variabilidad de la serie temporal.

Al extraer el componente aleatorio de la serie que se puede observar en la figura 3, se identifica que todos los puntos contienen aleatoriedad y eso es natural en las exportaciones de un país; sin embargo, al revisar los valores FOB en bruto del componente, se identifica que varios son despreciables debido a su valor-cantidad, en contraste con la serie original, por lo cual se aplica el cálculo de cuartiles al componente aleatorio y se califican como picos relevantes sobre el primer cuartil y bajo el $3^{\circ}$ cuartil, dada la naturaleza positiva y negativa de los mismos. En la figura 3 se puede observar el seccionamiento del componente aleatorio. 
Figura 3. Componente aleatorio de la serie de tiempo

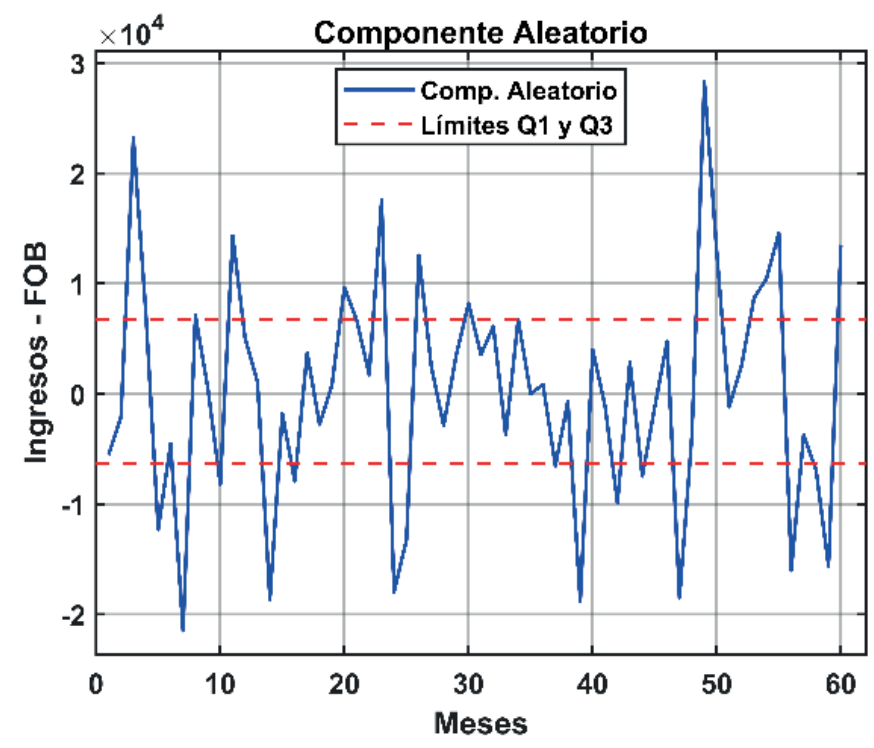

Fuente: elaboración propia.

Los picos resultantes (veintinueve) son extraídos del componente aleatorio para su clasificación y etiqueta temporal, pues en la siguiente fase del proyecto (cualitativa) son estudiados a fondo, como se puede ver en la siguiente sección.

\section{Análisis de picos e identificación de factores clave en exportaciones}

En la sección anterior los picos relevantes fueron identificados, clasificados y etiqueta- dos, en la presente sección cada uno de los veintinueve picos fueron analizados mediante investigación documental de organismos oficiales y producción científica asociada, permitiendo determinar la causa que dio origen al pico de ingresos FOB por exportación de banano y plátano en la serie temporal. Los ítems encontrados se pueden observar en la tabla 2.

Tabla 2. Ítems de causas de picos

\begin{tabular}{|l|l|l|}
\hline & \multicolumn{1}{|c|}{ Ítems en picos positivos } & \multicolumn{1}{c|}{ Ítems en picos negativos } \\
\hline 1 & Restricciones sanitarias. & Costos altos de producción y exportación. \\
\hline 2 & Variación de precio internacional. & Intenciones de acuerdo comerciales. \\
\hline 3 & Efectos del clima en países productores. & Altos costos, sin preferencias arancelarias. \\
\hline 4 & Impacto de ferias nacionales e internacionales. & Efectos del clima en países productores. \\
\hline 5 & Manejo y mejora de la producción. & Efecto de la competencia. \\
\hline 6 & Efecto de acuerdos comerciales. & Variación de precio internacional. \\
\hline 7 & Índice de productividad. & Variación de oferta/demanda. \\
\hline 8 & & Efectos económicos - apreciación moneda. \\
\hline
\end{tabular}

\footnotetext{
Fuente: elaboración propia.
} 
Si bien los ítems generales ya dan una pauta de las causas que provocan la variabilidad de ingresos FOB, también se presentan de diferente naturaleza en la serie, por lo cual se proceden a calificar mediante categorías, llamadas factores claves a gestionar dentro de las exportaciones ecuatorianas; estos factores son: político, económico, social, tecnológico, ambiental y productivo, con los cuales se procede a aplicar el proceso mencionado y a generar diagramas de Pareto, que se pueden identificar en las figuras 4 y 5.

Figura 4. Análisis picos positivos

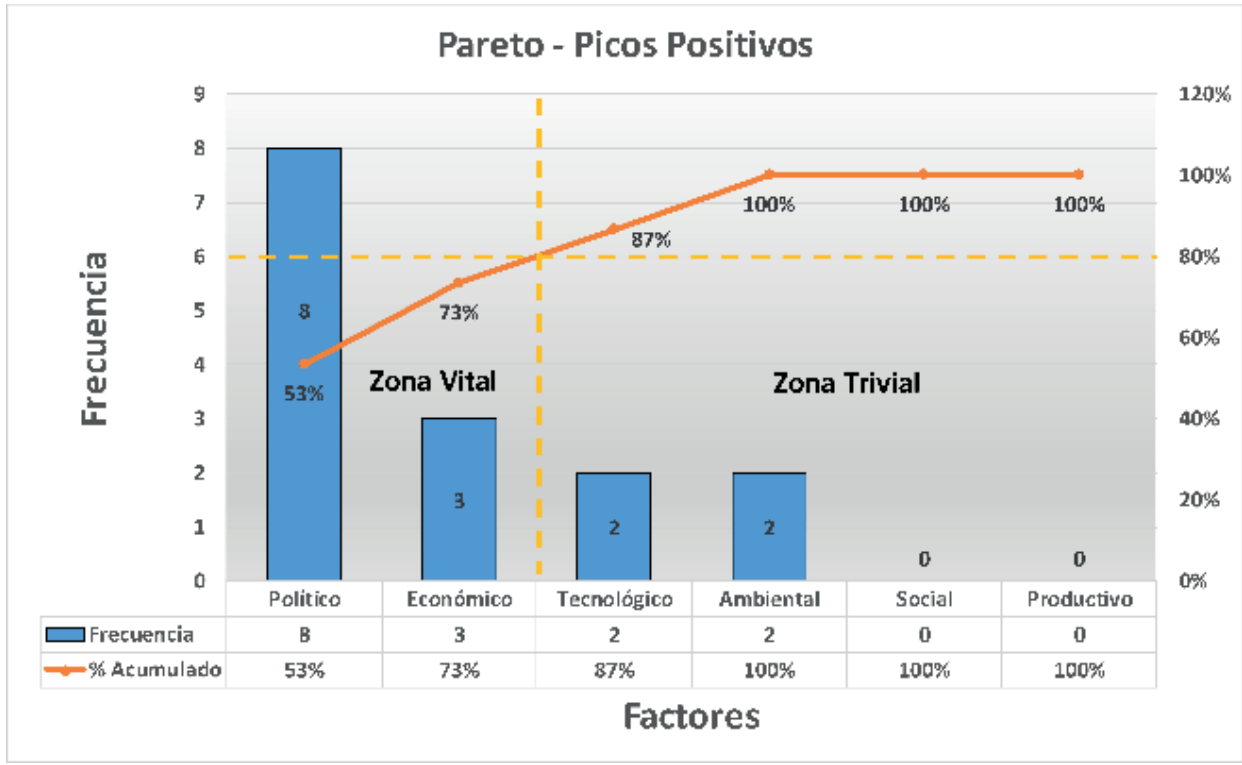

Fuente: elaboración propia

Figura 5. Análisis picos negativos

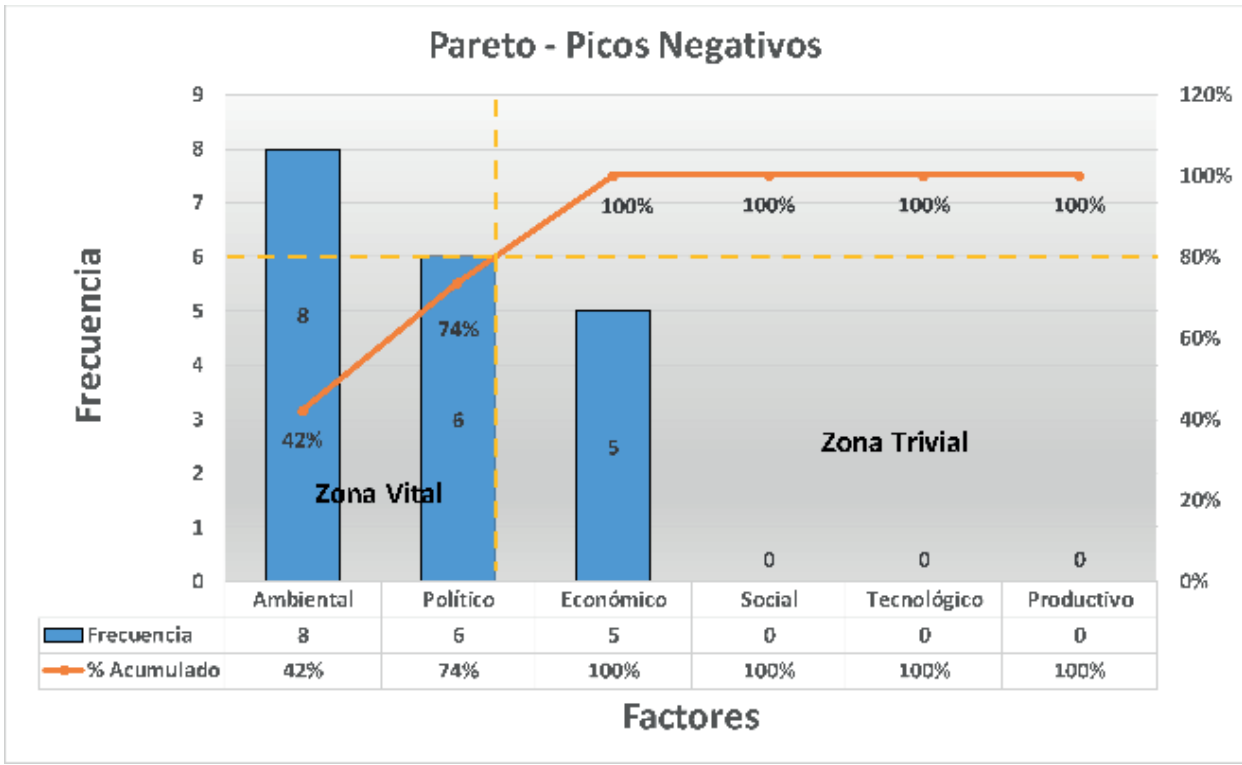

Fuente: elaboración propia. 
Con la aplicación de Pareto se puede identificar las zonas vitales y triviales asociadas a los factores relevantes del componente aleatorio, lo que permite determinar los componentes claves a gestionar, dentro de las exportaciones ecuatorianas de banano y plátano. Para el caso de picos positivos se puede observar que el $73 \%$ ha sido producido por factores políticos y económicos debiendo replicarse dichos eventos, dado que representan un incremento en ingresos al Estado por exportación de banano y plátano. Para el caso de picos negativos se determinó un $74 \%$ procedente de factores ambientales y políticos. En este escenario se debe tratar de evitar la réplica de dichos eventos, pues representan caídas relevantes en ingresos FOB por las exportaciones de banano y plátano.

\section{Conclusiones}

El banano y plátano a nivel mundial representan un importante commodity, por lo cual existen diversos estudios y constantes cambios en estrategias y procesos para su producción y comercialización. En el presente proyecto se logró determinar que la variabilidad positiva en ingresos FOB del Ecuador por exportaciones de banano y plátano, en los años 2013 a 2017, correspondieron a factores políticos con un $53 \%$ y económicos con un $20 \%$, los cuales sugieren replicar dichas prácticas o mejorar su periodicidad de ser el caso. Por otro lado, el decremento de dichos ingresos FOB, asociados a picos negativos relevantes, se produjeron en un 42 $\%$ por factores ambientales y en $32 \%$ por factores políticos, asociando estos picos a eventos que no se deben replicar o tratar de minimizarlos al máximo pues impactan en la balanza comercial ecuatoriana. Adicional a los factores relevantes se pudo identificar también diez casusas asociadas a variabilidad, que se repiten en el estudio de picos y que podrían ser base para estudios futuros de exportaciones de banano y plátano.

Para el caso del proceso de estudio de una serie temporal como la del presente proyecto, se determinó la necesidad de establecer previamente criterios relevantes que permitan seleccionar el método y parámetros de descomposición adecuados, obteniendo indicadores de ajuste idóneos, pues se calcularon los errores del porcentual absoluto medio con $3.6 \%$ y un porcentual medio de $-0.1 \%$.

\section{Referencias bibliográficas}

Andrade Rodríguez, P. L. y Meza Lino, A. D. (2017). Trade Agreement between Ecuador and the European Union: The case of the Ecuadorian banana sector [Acuerdo comercial entre Ecuador y la Unión Europea: El caso del sector bananero ecuatoriano]. Revista Espacios, 38(58), 26. Recuperado de http://ww.revistaespacios.com/ a17v38n58/17385826.html

Banco Central del Ecuador. (2018). Ecuador: evolución de la balanza comercial. Banco Central del Ecuador, febrero, 32. doi: 10.1017/СB09781107415324.004

Banco Central del Ecuador. (2020). Estadísticas del sector externo. Recuperado de https://contenido.bce.fin.ec/home1/estadisticas/bolmensual/IEMensual.jsp

Brassel, F., Breilh, J. y Zapat, A. (2011). ¿Agroindustria y soberanía alimentaria? : hacia una ley de agroindustria y empleo. Recuperado de http://biblioteca.clacso.edu.ar/Ecuador/sipae/20170627051014/pdf_427.pdf

Camino Mogro, S., Andrade Díaz, V. y Pesántez Villacís, D. (2016). Posicionamiento y eficiencia del banano, cacao y flores del Ecuador en el mercado mundial. Ciencia UNEMI, 9(19), 48-53.

Cleveland, R. (1990). STL: A Seasonal-Trend Descomposition Procedure Based on Loess. Journal of Official Statistics, 6(1), 3-73.

De la Cruz Guerrero, L. A. y Rosales Nieto, D. E. (2016). El control aduanero en el Ecuador, 
una visión al período 2013 - 2016. Economía y Negocios, 7(2), 25-37.

FAO. (2018). Banana Market Review Preliminary Results for 2018. Recuperado de http:// www.fao.org/fileadmin/templates/est/ COMM_MARKETS_MONITORING/Bananas/ Documents/Banana_Review_Update_December_2018.pdf

Fierro Ulloa, I. J. y Villacrés Rojas, C. E. (2014). Diagnóstico de la cadena logística de exportación del banano ecuatoriano hacia Estados Unidos de América. Saber, ciencia y libertad, 9(1), 77-90. doi: 10.18041/23823240/saber.2014v9n1.1985

Gujarati, D. (2007). Econometría (4.0 ed.). McGrawHill, Ed.

Hanke, J. y Wichern, A. (2010). Pronósticos en los negocios. México: Person Educación.

Jiménez Cabrera, J. H. (2018). Teoría de los juegos y su aplicación a los negocios internacionales: acuerdo comercial Unión Europea- Ecuador en el sector agrícola bananero. INNOVA Research Journal, 3(12), 55-66. https://doi.org/10.33890/innova. v3.n12.2018.768

León Serrano, L. A., Arcaya Sisalima, M. F., Barbotó Velásquez, N. A., \& Bermeo Pineda, Y. L. (2020). Ecuador: Análisis comparativo de las exportaciones de banano orgánico y convencional e incidencia en la Balanza Comercial, 2018. Revista Científica y Tecnológica UPSE, 7(2), 38-46. https://doi. org/10.26423/rctu.v7i2.521

Lind, D., Marchal, W., y Wathen, S. (2012). Estadística aplicada a los negocios y a la economía, 15 ed. México: McGraw-Hill
Martínez, H., y Peña, Y. (2005). La cadena del banano en Colombia. Una mirada global de su estructura y dinámica 1991-2005. In Ministerio Agricultura y Desarrollo Rural. Observatorio Agrocadenas Colombia (60).

Ministerio de Comercio Exterior. (2018). Informe mensual de comercio exterior - Ecuador.

Moreno Sarmiento, E. (2013). Predicción con series de tiempo y regresión. Panorama, 2(4), 36-58. doi: 10.15765/pnrm.v2i4.262

Murillo, J., Trejos, A. y Carvajal Olaya, P. (2003). Estudio del pronóstico de la demanda de energía eléctrica, utilizando modelos de series de tiempo, 3(23), 37-42. doi: $10.22517 / 23447214.7379$

Robinson, P. (2010). Responsible Retailing: The Practice of CSR in Banana Plantations in Costa Rica. Journal of Business Ethics, 91(2), 279-289.

Sanderson Bellamy, A. (2012). Banana Production Systems: Identification of Alternative Systems for More Sustainable Production. Royal Swedish Academy of Science, 42(3), 334-343.

The Observatory of Economic Complexity. (2020). Bananas. Recuperado el 19 de abril de 2020 de https://oec.world/en/profile/ hs92/20803/

Trujillo, D. J., Apunte, R. y Pereira, S. A. (2019). Ruido en exportaciones de cacao ecuatoriano a mercados internacionales. Espacios, 40, 30. Recuperado de http://www.revistaespacios.com/a19v40n30/19403003.html

Vásquez Orozco, R. (2010). El impacto del comercio del banano en el desarrollo del Ecuador. Afese temas internacionales, 53(53), 167-182. 